\title{
Une remarque sur la méthode des approximations successives dans la recherche des solutions périodiques des équations différentielles à paramètre retardé
}

\author{
by Z. MikokaJsKa (Kraków)
}

Nous avons démontré( $\left.{ }^{1}\right)$ l'existence d'une solution périodique de l'équation différo-différentielle

$$
x^{\prime}(t)=f(t, x(t), x(t-h)), \quad h>0
$$

dans le cas où $f(t, x, y)$ est continue et croissante par rapport à $x$ ou $y$, périodique par rapport à $t$ avec la période $h$. Dans la présente note nous démontrons une autre condition suffisante pour l'existence d'une solution (1) périodique, avec la période $T$ (qui peut être différente de $h$ ). Nous ne supposons plus que $f(t, x, y)$ est croissante par rapport à $x$ ou $y$, mais nous admettons qu'elle est de classe $C^{1}$ par rapport à $x, y$ et nous utilisons certaines évaluations de $\left|f_{x}\right|,\left|f_{y}\right|$ et $\left|f_{x}+f_{y}\right|$. La méthode des approximations successives dont nous nous servirons dans cette note est plus commode que celle employée dans $\left(^{1}\right)$.

1. Нтротніses H. 1. La fonction $f(t, x, y)$ est de classe $C^{1}$ par rapport $\grave{a}(x, y)$, continue par rapport $\dot{a}(t, x, y)$ et périodique par rapport $\dot{a} t$, de période $T>0$, pour tous les $(t, x, y)$.

2. Il existe une constante $m>0$ telle que pour chaque couple de fonctions périodiques $\sigma_{1}(t), \sigma_{2}(t)$ de période $T$ on a

$$
\begin{gathered}
\left|\int_{0}^{T}\left\{f_{x}\left(t, \sigma_{1}(t), \sigma_{2}(t)\right)+f_{y}\left(t, \sigma_{1}(t), \sigma_{2}(t)\right)\right\} d t\right| \geqslant m>0 \\
\left|f_{x}(t, x, y)\right| \leqslant M, \quad\left|f_{y}(t, x, y)\right| \leqslant M \\
\left|f_{x}(t, x, y)+f_{y}(t, x, y)\right| \leqslant N \\
\frac{M N T^{2}}{m}<1 .
\end{gathered}
$$

(') Z. Mikolajska, Sur l'existence d'une solution périodique d'une équation différentielle du premier ordre avec paramètre retardé, Ann. Polon. Math. 23 (1970), p. 25-36. 
THÉR丨̀ms 1. Sous les hypothèses $H$ il existe une solution périodique $x(t)$ de l'équation (1), de période $T$. La solution en question peut être obtenue comme la limite uniforme de la suite

$$
x_{n}(t)=a_{n}+\varphi_{n}(t),
$$

où les constantes $a_{n}$ et les fonctions $\varphi_{n}(t)$ sont definies par les relations suivantes:

$$
\varphi_{0}(t)=0 \quad \text { pour }-\infty<t<+\infty,
$$

$a_{0}$ étant la solution de l'équation numérique

$$
\int_{0}^{T} f\left(t, a_{0}, a_{0}\right) d t=0 .
$$

$D^{\prime}$ une façon analogue on definit $a_{n}$ et $\varphi_{n}(t)$

$$
\varphi_{n}(t)=\int_{0}^{t} f\left(s, a_{n-1}+\varphi_{n-1}(s), a_{n-1}+\varphi_{n-1}(s-h)\right) d s,
$$

$a_{n}$ est une solution de l'équation

$$
\int_{0}^{T} f\left(s, a_{n}+\varphi_{n}(s), a_{n}+\varphi_{n}(s-h)\right) d s=0 .
$$

Démonstration. Nous allons démontrer que:

I. Les fonctions $\varphi_{n}(t)$ sont périodiques de période $T$.

II. Pour chaque $n=0,1,2, \ldots$ l'équation (1.8) a une solution. unique $a_{n}$. $[0, T]$.

III. La suite $\left\{a_{n}, \varphi_{n}(t)\right\}$ converge uniformément dans l'intervalle

IV. La $\lim x_{n}(t)=x(t)$ est une solution périodique de l'équation (1).

Démonstration de $I . \varphi_{0}(t)$ est par définition périodique de période T. $f(t, x, y)$ étant périodique, en vertu de (1.6) et (1.7), $\varphi_{1}(t)$ est aussi périodique. Dans le cas où $\varphi_{n-1}(t)$ est périodique et $a_{n-1}$ satisfait à (1.8), la fonction $f$ étant périodique, $\varphi_{n}(t)$ définie par (1.7) l'est aussi. Dans le cas où tous les $a_{n}$ existent, $\varphi_{0}(t)$ étant périodique, tous les $\varphi_{n}(t)$ sont périodiques de période $T$.

Démonstration de II. Introduisons la notation suivante:

$$
\Gamma_{n}(a)=\int_{0}^{T} f\left[s, a+\varphi_{n}(s), a+\varphi_{n}(s-h)\right] d s .
$$

On a

$$
\Gamma_{n}^{\prime}(a)=\int_{0}^{T}\left\{f_{x}\left[8, a+\varphi_{n}(s), a+\varphi_{n}(s-h)\right]+f_{y}\left[s, a+\varphi_{n}(s), a+\varphi_{n}(s-h)\right]\right\} d s .
$$


En vertu de (1.1) on a $\left|\Gamma_{n}^{\prime}(a)\right| \geqslant T m>0$. Soit par exemple $\Gamma_{n}^{\prime}(a)$ $\geqslant T m>0$, d'où $\Gamma_{n}(a) \geqslant m a+\Gamma_{n}(0)$ pour $a>0, \Gamma_{n}(a) \leqslant m a+\Gamma_{n}(0)$ pour $a<0$ et par suite il existe un $R_{n}>0$ tel que $\Gamma_{n}\left(R_{n}\right)>0, \Gamma_{n}\left(-R_{n}\right)<0$ (pour $n=0,1,2, \ldots$ ).

De la continuité de $\Gamma_{n}(a)$ il vient qu'il existe un $a_{n} \epsilon\left(-R_{n}, R_{n}\right)$ tel que $\Gamma_{n}\left(a_{n}\right)=0$ pour $n=0,1,2, \ldots$

Démonstration de III. Pour démontrer la convergence de la suite $\left\{\varphi_{n}(t)\right\}$ envisageons la différence

$$
\begin{aligned}
& \left|\varphi_{n}(t)-\varphi_{n-1}(t)\right| \\
& =\mid \int_{0}^{t}\left\{f\left(s, a_{n-1}+\varphi_{n-1}(s), a_{n-1}+\varphi_{n-1}(s-h)\right)-\right. \\
& \left.\quad-f\left(s, a_{n-2}+\varphi_{n-2}(s), a_{n-2}+\varphi_{n-2}(s-h)\right)\right\} d s \mid \\
& =\mid \int_{0}^{t}\left\{f_{x}\left(s, \sigma_{n}(s), \vartheta_{n}(s)\right)\left[\left(\varphi_{n-1}(s)-q_{n-2}(s)\right)+\left(a_{n-1}-a_{n-2}\right)\right]+\right. \\
& \left.\quad+f_{v}\left(s, \sigma_{n}(s), \vartheta_{n}(s)\right)\left[\left(\varphi_{n-1}(s-h)-\varphi_{n-2}(s)\right)+\left(a_{n-1}-a_{n-2}\right)\right]\right\} d s \mid
\end{aligned}
$$

où les fonctions $\sigma_{n}(s), \vartheta_{n}(s)$, convenablement choisies, sont périodiques. En vertu de (1.8) on a

$$
\begin{aligned}
&\left(a_{n-1}-a_{n-2}\right) \int_{0}^{T} {\left[f_{x}\left(s, \sigma_{n}(s), \vartheta_{n}(s)\right)+f_{y}\left(s, \sigma_{n}(s), \vartheta_{n}(s)\right)\right] d s } \\
&=-\int_{0}^{T} f_{x}\left(s, \sigma_{n}(s), \vartheta_{n}(s)\right)\left(\varphi_{n-1}(s)-\varphi_{n-2}(s)\right) d s- \\
& \quad-\int_{0}^{T} f_{y}\left(s, \sigma_{n}(s), \vartheta_{n}(s)\right)\left(\varphi_{n-1}(s-h)-\varphi_{n-2}(s-h)\right) d s,
\end{aligned}
$$

d'où

$$
\begin{aligned}
& \left|\varphi_{n}(t)-\varphi_{n-1}(t)\right|=\mid\left[1-\frac{\int_{0}^{t}\left(f_{x}\left(s, \sigma_{n}, \vartheta_{n}\right)+f_{y}\left(s, \sigma_{n}, \vartheta_{n}\right)\right) d s}{\int_{0}^{T}\left(f_{x}\left(s, \sigma_{n}, \vartheta_{n}\right)+f_{y}\left(s, \sigma_{n}, \vartheta_{n}\right)\right) d s}\right\rfloor \times \\
& \times\left\{\int_{0}^{t} f_{x}\left(s, \sigma_{n}, \vartheta_{n}\right)\left(\varphi_{n-1}(s)-\varphi_{n-2}(s)\right) d s+\right. \\
& \left.+\int_{0}^{t} f_{\nu}\left(s, \sigma_{n}, \vartheta_{n}\right)\left(\varphi_{n-1}(s-h)-\varphi_{n-2}(s-h)\right) d s\right\}-
\end{aligned}
$$




$$
\begin{aligned}
& -\frac{\int_{0}^{t}\left\{f_{x}\left(s, \sigma_{n}, \vartheta_{n}\right)+f_{y}\left(s, \sigma_{n}, \vartheta_{n}\right)\right] d s}{\int_{0}^{T}\left[f_{x}\left(s, \sigma_{n}, \vartheta_{n}\right)+f_{y}\left(s, \sigma_{n}, \vartheta^{n}\right)\right] d s} \int_{i}^{T}\left\{f_{x}\left(s, \sigma_{n}, \vartheta_{n}\right)\left(\varphi_{n-1}(s)-\varphi_{n-2}(s)\right)+\right. \\
& \left.\quad+f_{y}\left(s, \sigma_{n}, \vartheta_{n}\right)\left(\varphi_{n-1}(s-h)-\varphi_{n-2}(s-h)\right)\right\} d s \mid \\
& \leqslant\left|\frac{\int_{t}^{T}\left[f_{x}+f_{y}\right] d s}{\int_{0}^{T}\left[f_{x}+f_{y}\right] d s}\right| \int_{0}^{t}\left\{\left|f_{x}\right|\left|\varphi_{n-1}(s)-\varphi_{n-2}(s)\right|+\left|f_{y}\right| \mid \varphi_{n-1}(s-h)-\right. \\
& \left.-\varphi_{n-2}(s-h) \mid\right\} d s+\left|\frac{\int_{0}^{t}\left(f_{x}+f_{y}\right) d s}{\int_{0}^{T}\left(f_{x}+f_{y}\right) d s}\right| \int_{i}^{T}\left\{\left|f_{x}\right|\left|\varphi_{n-1}(s)-\varphi_{n-2}(s)\right|+\right. \\
& \left.\left|f_{y}\right|\left|\varphi_{n-1}(s-h)-\varphi_{n-2}(s-h)\right|\right\} d s .
\end{aligned}
$$

En vertu de (1.2) on a

$$
\begin{aligned}
\left|\varphi_{n}(t)-\varphi_{n-1}(t)\right| \leqslant \frac{2 M N}{m}((T-t) t+t(T-t)) \varrho_{n-1}= & \frac{4 M N}{m} \varrho_{n-1}(T-t) t \\
& \cdot \text { pour } 0<t<T,
\end{aligned}
$$

où $\varrho_{n}=\max \left|\varphi_{n}(s)-\varphi_{n-1}(s)\right|$ pour $0 \leqslant t \leqslant T$. On vérifie facilement que le maximum de la fonction $(T-t) t$ dans l'intervalle $[0, T]$ est égal à $\left(T-\frac{1}{2} T\right) \frac{T}{2}=\frac{T^{2}}{4}$, d'où l'on tire

$$
\varrho_{n} \leqslant \frac{M N T^{2}}{m} \varrho_{n-1}, \quad\left|a_{n}-a_{n-1}\right| \leqslant \frac{2 M T}{m} \varrho_{n}
$$

De là, en vertu de (1.3), on obtient la convergence

et par suite

$$
\varphi_{n}(t) \rightarrow \varphi(t) \quad \text { pour } 0 \leqslant t \leqslant T, a_{n} \rightarrow a,
$$

$$
x_{n}(t)=a_{n}+\varphi_{n}(t) \rightarrow a+\varphi(t)=x(t) .
$$

La convergence obtenue est uniforme dans l'intervalle $[0, T]$. Les fonctions $\varphi_{n}(t)$ étant périodiques, la convergence uniforme a lieu dans tout l'intervalle $(-\infty,+\infty)$ et la fonction obtenue est périodique.

La démonstration de IV résulte immédiatement de la définition (1.4), de (1.7), de la convergence uniforme de $\varphi_{n}$ et de la convergence de $a_{n}$. La démonstration du théorème 1 est ainsi terminée. 
2. Remarque 1. Il est évident que dans la définition de la suite $\varphi_{n}$ on peut remplacer la fonction $\varphi_{0}(t)=0$ par une fonction quelconque périodique et continue $\bar{\varphi}(t)$.

Remarque 2. Dans le cas où $T=h$ les conditions (1.2) et (1.3) peuvent être remplacées par des conditions moins restrictives. Dans le cas envisagé on a le théorème suivant:

HYPothises $\bar{H}_{1}$. 1. La fonction $f(t, x, y)$ est de classe $C^{1}$ par rapport $\grave{a}(x, y)$, continue par rapport $\grave{a}(t, x, y)$ et périodique par rapport $\grave{a} t$, de période $T=h$.

2. Il existe une constante $m>0$ telle que pour chaque couple de fonctions $\sigma_{1}(t), \sigma_{2}(t)$ périodique de période $h$ on a

$$
\begin{gathered}
\left|\int_{0}^{h}\left\{f_{x}\left(s, \sigma_{1}(s), \sigma_{2}(s)\right)+f_{y}\left(s, \sigma_{1}(s), \sigma_{2}(s)\right)\right\} d s\right| \geqslant m>0 \\
\left|f_{x}(s, x, y)+f_{y}(s, x, y)\right| \leqslant N \\
\frac{(N h)^{2}}{2 m}<1
\end{gathered}
$$

Thíorìme 2. Sous les hypothèses $\overline{\mathrm{H}}$ il existe une solution périodique $x(t)$ de l'équation (1) de période $h$.

Démonstration. On a, en vertu de la périodicité de $\varphi_{n}(t)$,

$$
\begin{aligned}
\left|\varphi_{n}(t)-\varphi_{n-1}(t)\right| \leqslant & \int_{0}^{t}\left\{f_{x}\left(s, \sigma_{n}, \vartheta_{n}\right)+f_{y}\left(s, \sigma_{n}, \vartheta_{n}\right)\left(\varphi_{n-1}(s)-\right.\right. \\
& \left.\left.-\varphi_{n-2}(s)\right)\right\} d s\left|\frac{\int_{t}^{T}\left(f_{x}+f_{y}\right) d s}{\int_{0}^{T}\left(f_{x}+f_{y}\right) d s}\right|+ \\
& +\left|\frac{\int_{0}^{t}\left(f_{x}+f_{y}\right) d s}{T}\right|\left|\int_{0}^{T}\left(f_{x}+f_{y}\right)\left(f_{n-1}(s)-\varphi_{n-2}(s)\right) d s\right| \\
\leqslant & \frac{2 N^{2}}{m} \varrho_{n-1}(T-t) t \leqslant \frac{(N T)^{2}}{m} \varrho_{n-1}=\frac{(N h)^{2}}{m} \varrho_{n-1}
\end{aligned}
$$

et

$$
\left|a_{n-1}-a_{n-2}\right| \leqslant \frac{N h}{m} \varrho_{n-1} .
$$

3. Remarque 3. Chaque solution périodique de l'équation (1) peut être obtenue comme la limite d'une suite de la forme (1.4), où $a_{n}$ 
et $\varphi_{n}(t)$ sont définies par les relations (1.7) et (1.8) pour $n=1,2, \ldots$ Pour obtenir la solution $x(t)$ il suffit de poser

$$
a_{0}=x(0), \varphi_{0}(t)=\int_{0}^{t} f(s, x(s), x(s-h)) d s .
$$

On obtient $a_{n}=a_{0}, \varphi_{n}(t)-\varphi_{0}(t)$ pour $n=1,2, \ldots$ et par suite $x_{n}(t) \rightarrow x(t)$ uniformément.

4. Thtorìme 3. Dans les hypothèses H chaque solution périodique de l'équation (1) est égale à la solution $x(t)$ définie dans la démonstration du théorème 1: $x(t)=a+\varphi(t)$, c'est-à-dire il existe une solution unique périodique de période $T$.

Démonstration. Envisageons une solution périodique $w(t)$ de l'équation (1). De la remarque 3 il vient qu'il existe une fonction $\tilde{\varphi}_{0}(t)$ périodique de période $T$ telle que la suite $w_{n}(t)=\tilde{a}_{n}+\tilde{\varphi}_{n}(t)$ où $\tilde{a}_{n}$ et $\tilde{\varphi}_{n}(t)$ sont définis par (1.7) et (1.8) avec $\varphi_{0}(t)=\tilde{\varphi}_{0}(t)$ (au lieu de $\varphi_{0}(t)=0$ ). On vérifie facilement que

$$
\left|\tilde{\varphi}_{n}(t)-\varphi_{n}(t)\right| \leqslant \frac{M N T}{m} \tilde{\varrho}_{n-1} \leqslant \tilde{\varrho}_{0}\left(\frac{N M T}{m}\right)^{n},
$$

où $\tilde{\varrho}_{n}=\max \left|\tilde{\varphi}_{n}(t)-\varphi_{n}(t)\right|$. On a donc

$$
\tilde{\varrho}_{n} \leqslant \tilde{\varrho}_{0}\left(\frac{N M T}{m}\right)^{n}
$$

et par suite $\tilde{\varrho}_{n} \rightarrow 0$. On vérifie facilement que l'on a aussi $\left|\tilde{a}_{n}-a_{n}\right| \rightarrow 0$ et par suite $\tilde{\varphi}(t)=\varphi(t), \tilde{a}=a$ et $w(t)=x(t)$.

Remarque 4. Les hypothèses (1.1) et (1.3) du théorème 1 sont indispensables. Dans le cas où $\left[f_{x}+f_{y}\right]$ peut être égal à zéro on peut poser $f_{x}=f_{y}=0$ et $f(t, x, y)=c>0$. Une telle fonction est périodique et elle satisfait à (1.2) où $M>0, N>0$ sont quelconques. Cependant il n'existe pas de solutions périodiques de l'équation $x^{\prime}(t)=c>0$.

5. 'Remarque 5. Dans la suite nous construirons un exemple d'une équation de la forme (1) satisfaisant aux hypothèses 1 et (1.1), (1.2), mais telle qu'il n'existe pas de solution périodique de l'équation envisagée, c'est-à-dire nous démontrons que l'hypothèse (1.3) est indispensable.

ExEMPLE. Envisageons une équation de la forme

$$
x^{\prime}(t)=p(t) x(t)+q(t, x(t-h))+r(t)
$$

où $h$ est une constante positive $h>0$ (quelconque) et les fonctions $p(t)$, 
$q(t, y), r(t)$ sont continues et périodiques par rapport à $t$ de période $T=4 h$ et satisfont aux conditions suivantes

$$
\begin{gathered}
p(t)>0 \quad \text { pour } 0<t<h, \\
p(t) \equiv 0 \quad \text { pour } h \leqslant t \leqslant T=4 h \\
p(t+n T)=p(t) \quad \text { pour } n=1,2,3, \ldots, \\
\int_{0}^{h} p(t) d t=c=\ln \frac{1-\varepsilon}{\varepsilon}
\end{gathered}
$$

où $\varepsilon$ est une constante positive

$$
0<\varepsilon<\frac{1}{1+e^{2}}<\frac{1}{2}
$$

$$
q(t, y)= \begin{cases}-k p(t-h) y & \text { pour } h \leqslant t \leqslant 2 h,-\infty<y<+\infty \\ \varepsilon p(t-2 h) y & \text { pour } 2 h \leqslant t \leqslant 3 h, y \geqslant 0 \\ \frac{\varepsilon}{2} p(t-2 h)(y+1)^{2} & \text { pour } 2 h \leqslant t \leqslant 3 h,-1 \leqslant y \leqslant 0 \\ 0 & \text { pour } 2 h \leqslant t \leqslant 3 h, y \leqslant-1 \\ 0 & \text { pour } 3 h \leqslant t \leqslant 4 h=T \\ 0 & \text { pour } 0 \leqslant t \leqslant h\end{cases}
$$

Dans (5.7) posons

$$
r(t)= \begin{cases}0 & \text { pour } 0 \leqslant t \leqslant 3 h, \\ p(t-3 h) & \text { pour } 3 h \leqslant t \leqslant 4 h .\end{cases}
$$

$$
k=\frac{1-\varepsilon}{1-2 \varepsilon}
$$

Démontrons que dans le cas envisagé il n'existe pas de solutions périodiques de l'équation (5.1) de période $T=4 h$.

Envisageons une solution quelconque $x(t)$ de l'équation (5.1) et posons par définition

$$
x_{0}=x(0) \text {. }
$$

Dans l'intervalle $0 \leqslant t \leqslant h$ nous avons, en vertu de (5.7) et (5.8),

et par suite

$$
x^{\prime}(t)=p(t) x(t) \quad \text { pour } 0 \leqslant t \leqslant h
$$

$$
\begin{gathered}
x(t)=x_{0} \exp \int_{0}^{t} p(s) d s \quad \text { pour } 0 \leqslant t \leqslant h, \\
x(h)=x_{0} \exp \int_{0}^{h} p(s) d s=x_{0} e^{c}=x_{0} \frac{1-\varepsilon}{\varepsilon} .
\end{gathered}
$$


Dans l'intervalle $h \leqslant t \leqslant 2 h$ nous avons, en vertu de (5.3) et (5.8),

$$
x^{\prime}(t)=-k p(t-h) x(t-h)=\frac{1-\varepsilon}{1-2 \varepsilon} p(t-h) x(t-h)
$$

d'où, en vertu de (5.10), (5.11) et (5.9), il vient

$$
\begin{aligned}
x(t) & =x_{0} \frac{1-\varepsilon}{\varepsilon}-\frac{1-\varepsilon}{1-2 \varepsilon} x_{0} \int_{h}^{t} p(\tau+h) \exp \int_{0}^{\tau-h} p(s) d s d \tau \\
& =x_{0}\left\{\frac{1-\varepsilon}{\varepsilon}-\frac{1-\varepsilon}{1-2 \varepsilon} \int_{0}^{t-h} p(\tau) \exp \int_{0}^{\tau} p(s) d s d \tau\right\} \\
& =x_{0}(1-\varepsilon)\left\{\frac{1}{\varepsilon}-\frac{1}{1-2 \varepsilon}\left[e^{\substack{t-h \\
f p(s) d s}}-1\right]\right\} .
\end{aligned}
$$

De là on obtient pour $t=2 h$,

$$
\begin{aligned}
x(2 h) & =x_{0}(1-\varepsilon)\left\{\frac{1}{\varepsilon}-\frac{1}{1-2 \varepsilon}\left[e^{c}-1\right]\right\} \\
& =x_{0}(1-\varepsilon) \frac{1}{\varepsilon}\left[1-\frac{1}{1-2 \varepsilon}(1-2 \varepsilon)\right]=0,
\end{aligned}
$$

c'est-à-dire que pour chaque solution de l'équation (5.1) définie dans tout l'intervalle $0 \leqslant t \leqslant T$ on a $x(2 h)=0$ et dans l'intervalle $[0,2 h$ ) on a

$$
\begin{array}{ll}
x(t)>0 & \text { pour } x_{0}>0 \\
x(t) \equiv 0 & \text { pour } x_{0}=0 \\
x(t)<0 & \text { pour } x_{0}<0
\end{array}
$$

Envisageons le cas $x_{0}>0$. Dans l'intervalle [2h,3h] nous avons $x(t-h)>0$ et par suite

$$
\begin{gathered}
x^{\prime}(t)=\varepsilon p(t-2 h) x(t-h) \quad \text { pour } 2 h \leqslant t \leqslant 3 h \\
x(t)=x_{0} \frac{1-\varepsilon}{\varepsilon(1-2 \varepsilon)} \int_{2 h}^{t}\left\{(1-\varepsilon)-\varepsilon\left[\exp \int_{0}^{\tau-2 h} p(s) d s\right]\right\} p(\tau-2 h) d \tau \\
=\frac{(1-\varepsilon) x_{0}}{\varepsilon(1-2 \varepsilon)}\left\{(1-\varepsilon) \int_{0}^{t-2 h} p(s) d s-\varepsilon\left[\exp \int_{0}^{t-2 h} p(s) d s-1\right]\right\} .
\end{gathered}
$$


De (5.5) il vient

$$
\begin{aligned}
x(3 h) & =\frac{(1-\varepsilon) x_{0}}{\varepsilon(1-2 \varepsilon)}\left\{(1-\varepsilon) \ln \frac{1-\varepsilon}{\varepsilon}+\varepsilon-\varepsilon \frac{1-\varepsilon}{\varepsilon}\right\} \\
& =\frac{(1-\varepsilon) x_{0}}{1-2 \varepsilon}\left\{\frac{1-\varepsilon}{\varepsilon}\left(\ln \frac{1-\varepsilon}{\varepsilon}-1\right)+1\right\} .
\end{aligned}
$$

Posons

$$
\varepsilon<\frac{1}{1+e^{2}}<\frac{1}{2}
$$

Nous obtenons

$$
\frac{1-\varepsilon}{\varepsilon}>\frac{1+e^{2}}{1+e^{2}} e^{2}=e^{2}>1
$$

et par suite

$$
x(3 h)>k x_{0}\left\{\left(\ln e^{2}-1\right) e^{2}+1\right\}=k x_{0}\left(e^{2}+1\right) .
$$

En vertu de (5.12) on a

$$
\varepsilon<\frac{1}{1+e^{2}}=\frac{e^{2}-1}{e^{4}-1}<e^{2} \frac{\left(e^{2}+1\right)}{e^{4}-1}=\frac{e^{2}}{e^{2}-1}
$$

et par suite

$$
\left(\frac{2}{e^{2}+1}-1\right) \varepsilon=\frac{1-e^{2}}{1+e^{2}} \varepsilon>\frac{e^{2}}{e^{2}-1} \cdot \frac{1-e^{2}}{1+e^{2}}=\frac{1}{e^{2}+1}-1
$$

d'où on a

$$
\begin{gathered}
1-\varepsilon>\frac{1}{e^{\varepsilon}+1}-\frac{2 \varepsilon}{e^{2}+1}=\frac{1-2 \varepsilon}{e^{2}+1}, \quad 2 \varepsilon<1 ; \\
\frac{1-\varepsilon}{1-2 \varepsilon}>\frac{1}{e^{2}+1} .
\end{gathered}
$$

Donc, en vertu de (5.13) et (5:9),

$$
x(3 h)>\frac{1-\varepsilon}{1-2 \varepsilon}\left(e^{2}+1\right) x_{0}>x_{0} .
$$

Pour $3 h \leqslant t \leqslant T=4 h$ (cf. (5.8) et (5.1)),

et

$$
x^{\prime}(t)=p(t-3 h)
$$

$$
\begin{gathered}
x(t)=x(3 h)+\int_{3 h}^{t} p(s-3 h) d s=x(3 h)+\int_{0}^{t-3 h} p(s) d s, \\
x(T)=x(4 h)=x(3 h)+\int_{0}^{h} p(s) d s=x(3 h)+c>x_{0}+c>x_{0},
\end{gathered}
$$


d'où il vient que pour chaque $x_{0}>0$ la solution $x(t)$ de l'équation en: visagée n'est pas périodique. Pour $x_{0}=0$ aussi la solution $x(t)$ n'est pas périodique. Elle est égale à 0 dans l'intervalle $0 \leqslant t \leqslant 3 h$ et $\int_{0}^{t-3 h} p(s) d s$ pour $3 h \leqslant t \leqslant 4 h=T$, d'où $x(T)=\int_{0}^{h} p(s) d s=c>0=x(0)$.

Pour $x_{0} \leqslant 0$ on a l'équation et

$$
x^{\prime}(t)=0 \quad \text { pour tout } t \epsilon[2 h, 3 h] \text { telle que } x(t) \leqslant-1
$$

$$
x^{\prime}(t)=\frac{\varepsilon}{2} p(t-2 h)(x(t-h)+1)^{2}>0
$$$$
\text { pour } t \text { tels que } 0>x(t-h)>-1,2 h \leqslant t \leqslant 3 h \text {, }
$$

c'est-à-dire

$$
x(t)>x(2 h)=0 \quad \text { pour } 2 h<t \leqslant 3 h .
$$

Dans l'intervalle $3 h \leqslant t \leqslant 4 h$

et par suite

$$
x^{\prime}(t)=p(t-3 h) \geqslant 0 \quad \text { pour } 3 h \leqslant t \leqslant 4 h
$$

$$
x(T) \geqslant x(3 h)>0>x_{0} .
$$

Ainsi nous avons démontré que $x(t)$ n'est périodique ni pour $x_{0} \geqslant 0$ ni pour $x_{0}<0$. I reste a prouver que la fonction

$$
f(t, x, y)=p(t) x+q(t, y)+r(t)
$$

satisfait aux conditions 1 ; (1.1) et (1.2). La condition 1 est évidente. Envisageons $f_{x}+f_{v}$,

$$
\begin{gathered}
f_{x}(t, x, y)=p(t), \quad f_{y}(t, x, y)=q_{y}(t, y), \\
\int_{0}^{T}\left\{f_{x}\left(t, \sigma_{1}, \sigma_{2}\right)+f_{y}\left(t, \sigma_{1}, \sigma_{2}\right)\right\} d t=\int_{0}^{h} p(s) d s-k \int_{0}^{h} p(s) d s+\int_{2 h}^{3 h} q_{y}\left(s, \sigma_{2}\right) d s \\
\leqslant \int_{0}^{h} p(s) d s(1-k+\varepsilon)=\left(1-\frac{1-\varepsilon}{1-2 \varepsilon}+\varepsilon\right) c=\frac{-2 \varepsilon^{2}}{1-2 \varepsilon} c
\end{gathered}
$$

et par suite

$$
\left|\int_{0}^{T} f_{x}\left(s, \sigma_{1}, \sigma_{2}\right)+f_{y}\left(s, \sigma_{1}, \sigma_{2}\right) d s\right| \geqslant \frac{2 \varepsilon^{2}}{1-2 \varepsilon} c>0
$$

pour chaque couple de fonctions périodiques $\sigma_{1}(t)$ et $\sigma_{2}(t)$. 